\title{
Abnormal elevation of myocardial necrosis biomarkers after coronary artery bypass grafting without established myocardial infarction assessed by cardiac magnetic resonance
}

\author{
Fernando Teiichi Costa Oikawa, Whady Hueb ${ }^{*}$ (D), Cesar Higa Nomura, Alexandre Ciappina Hueb, \\ Alexandre Volney Villa, Leandro Menezes Alves da Costa, Rodrigo Morel Vieira de Melo, Paulo Cury Rezende, \\ Carlos Alexandre Wainrober Segre, Cibele Larrosa Garzillo, Eduardo Gomes Lima, \\ Jose Antonio Franchini Ramires and Roberto Kalil Filho
}

\begin{abstract}
Background: The diagnosis of peri-procedural myocardial infarction is complex, especially after the emergence of high-sensitivity markers of myocardial necrosis.

Methods: In this study, patients with normal baseline cardiac biomarkers and formal indication for elective onpump coronary bypass surgery were evaluated. Electrocardiograms, cardiac biomarkers, and cardiac magnetic resonance imaging with late gadolinium enhancement were performed before and after procedures. Myocardial infarction was defined as more than ten times the upper reference limit of the 99th percentile for troponin I and for creatine kinase isoform (CK-MB) and by the findings of new late gadolinium enhancement on cardiac magnetic resonance. We assessed the release of cardiac biomarkers in patients with no evidence of myocardial infarction on cardiac magnetic resonance.

Results: Of 75 patients referred for on-pump coronary bypass surgery, 54 (100\%) did not have evidence of myocardial infarction on cardiac magnetic resonance. However, all had a peak troponin I above the 99th percentile; 52 (96\%) had an elevation 10 times higher than the 99th percentile. Regarding CK-MB, 54 (100\%) patients had a peak CK-MB above the 99th percentile limit, and only 13 (24\%) had an elevation greater than 10 times the 99th percentile. The median value of troponin I peak was 3.15 (1.2 to 3.9) ng/mL, which represented 78.7 times the 99th percentile.

Conclusion: In this study, different from CK-MB findings, troponin was significantly increased in the absence of myocardial infarction on cardiac magnetic resonance. Thus, CK-MB was more accurate than troponin I for excluding procedure-related myocardial infarction. These data suggest a higher troponin cutoff for the diagnosis of coronary bypass surgery related myocardial infarction.
\end{abstract}

Clinical trial registration: http://www.isrctn.com/ISRCTN09454308. Registered 08 May 2012.

Keywords: Myocardial infarction, Biomarkers, Coronary bypass surgery, Periprocedural, Troponin

\footnotetext{
* Correspondence: mass@incor.usp.br

Instituto do Coracao (InCor), Hospital das Clinicas HCFMUSP, Faculdade de

Medicina, Universidade de São Paulo, São Paulo, SP, Brazil
} 


\section{Background}

Myocardial necrosis biomarkers are frequently elevated after cardiac revascularization procedures. However, the diagnosis of acute myocardial infarction (MI) after a revascularization procedure is still a controversial issue. This inability to diagnose MI makes it more difficult to establish a specific therapeutic strategy. With the appearance of high-sensitivity troponins, a myriad of false-positive diagnoses for myocardial infarction have emerged. In 2000 and 2007 in an attempt to standardize the criteria for diagnosing MI, the European Society of Cardiology, the American College of Cardiology, the American Heart Association, and the World Heart Federation formed a joint task force to address this issue, but the task force was unable to make a satisfactory decision. Therefore, the problem still remained. To reduce diagnostic mistakes, in 2012, this same group arbitrarily raised the cutoff point to 10 times the 99th percentile, but with no solid scientific basis for doing so [1]. Troponin (cTnI) and the creatine kinase isoform (CK-MB) do not reflect, alone, the occurrence of MI related to occlusion of the graft or native artery or varying degrees of myocardial injury. Release of myocardial necrosis markers may be related to incomplete myocardial protection; reperfusion injury; a systemic inflammatory state, including inevitable postsurgical trauma; the handling of intramyocardial vessels; and cardiac defibrillator use $[2,3]$. Cardiac troponin may also be increased when nonsurgical damage is present, such as sepsis and thromboembolic phenomena [1]. cTnIs have also been found elevated in athletes after marathons [4]. This makes the identification of small areas of injury very difficult to assess in clinical practice [5].

Parallel to the increased sensitivity of troponin assays, imaging methods have achieved better accuracy for exclusion of the diagnosis of myocardial infarction. Thus, due to the limitations on the interpretation of biomarkers after coronary artery bypass grafting (CABG) and the difficulty of excluding MI, cardiac magnetic resonance imaging (CMR) has enabled a more detailed evaluation of the myocardium.

Therefore, in this study, we aimed to examine the release of biomarkers after CABG in patients with no evidence of late enhancement on CMR.

\section{Patients and methods}

Details of the study design, protocol, patient selection, and inclusion criteria have been previously reported [6]. Briefly, patients with preserved left ventricular function and angiographic coronary artery stenosis of more than $70 \%$ confirmed by a visually reviewed document, and with multiple-vessel involvement, and documented ischemia were included. Stress testing or evaluation of stable angina according to the Canadian Cardiovascular Society guidelines (Class II or III) established the presence of ischemia.
All patients were candidates for on-pump coronary artery bypass grafting (ONCAB). Patients were excluded if they had undergone any previous mechanical interventions, and had experienced recent thromboembolic events, systemic inflammatory disease, or kidney failure.

\section{Trial outcomes}

The primary outcome was the occurrence of MI based on the release of the biomarkers, cTnI and CK-MB, in patients with no late gadolinium enhancement (LGE) assessed by CMR.

\section{Methods}

\section{Surgical technique}

In accordance with current best practices, the same team of surgeons with experience in ONCAB performed the procedures. Surgical access to the heart was through a standard median sternotomy in all cases. All incisions and closure techniques were performed in the same way in all patients to limit variability among patients.

\section{CMR protocol}

CMR was performed before and after the surgical procedure. CMR, considered the gold standard, allows highprecision assessment that is reproducible in the same test. Recent studies indicate that CMR detects MI very accurately and provides results similar or superior to results with radionuclide imaging [7-10].

All patients underwent CMR 2 days before the intervention and 6 days after each invasive procedure during the hospitalization period. A 1.5-T Achieva Magnetic Resonance scanner (Philips Healthcare, Andover, MA) was used. Steady-state free precession cine images were acquired in 2 long-axis ( 2 and 4 chambers) views and 8 to 10 short-axis views of the left ventricle. Contrastenhanced images were acquired in long- and short-axis planes identical to the cine images. Typical voxel size was $1.6 \times 2.1 \times 8 \mathrm{~mm}$, with a reconstruction matrix of 528 and a reconstructed voxel size of $0.6 \mathrm{~mm}$. The method for acquiring and analyzing CMR was standardized in our service and was reproduced according to conventional techniques [11, 12]. Delayed enhancement on CMR was performed with a phase-sensitive inversion recovery (PSIR) sequence (repetition time $6.1 \mathrm{~ms}$ echo time $3.0 \mathrm{~ms}$, voxel size $1.6 \times 2.1 \times 8 \mathrm{~mm}$, flip angle $25^{\circ}$ ) following a 5-min time delay after the administration of $0.1 \mathrm{mmol} / \mathrm{kg}$ contrast agent (GadoteratemeglumineGdDOTA $^{\mathrm{Tm}}$, Guerbet SA, France). Images were acquired in 2 long-axis planes and in a short-axis stack covering the entire left ventricle. The inversion time was meticulously adjusted throughout the acquisition to obtain optimal nulling of remote normal myocardium. The slice thickness at the apex was reduced to $5 \mathrm{~mm}$ to avoid a partial volume effect. MI was defined as the identification of 
hyper-enhancement in the myocardium on CMR. Infarcted regions exhibit this phenomenon, which might be due to an increased volume of distribution of the contrast agent, because of rupture of myocyte membranes and slow contrast washout [10].

\section{CMR analysis}

All areas of late gadolinium-diethylene-triamine-pentaacetic acid (DTPA) hyper-enhancement were quantified by 2 experienced observers who interpreted the LGE while blinded to the interventional technique and biochemical data. When measurements differed, a third observer performed a review, and a consensus was obtained. Hyper-enhanced pixels were defined as those with image intensities exceeding 2 standard deviations greater than the mean of image intensities in a remote myocardial region in the same image. Pre-intervention and post-intervention scans were read side by side in both surgical techniques, with and without extracorporeal circulation.

\section{Biochemistry}

All blood samples for measurement of cTnI and CK$\mathrm{MB}$ were collected immediately before and 6, 12, 24, 36,48 , and $72 \mathrm{~h}$ after on-pump CABG. The surgeon and clinical team were blinded to the CK-MB or cTnI data. All samples were centrifuged at $3000 \mathrm{rpm}$ for $20 \mathrm{~min}$ and analyzed within $2 \mathrm{~h}$ after collection. Analyses of cTnI and CK-MB were performed using an ADVIA Centaur immunoassay analyzer (Siemens Health Care Diagnostics, Tarrytown, NY). According to the manufacturer, the lower limit of detection of cTnI using the high-sensitivity Ultra kit is $0.006 \mathrm{ng} / \mathrm{mL}$, and the 99th percentile upper reference limit (URL) is $0.04 \mathrm{ng} / \mathrm{mL}$. The assay precision represented by the percentage coefficient of variation is $10 \%$ at $0.03 \mathrm{ng} /$ $\mathrm{mL}$. The detection limit of the CK-MB mass kit (Acute Care $^{\mathrm{rn}}$ CK-MB assay Siemens ${ }^{\mathrm{nw}}$ ) is $0.18 \mathrm{ng} / \mathrm{mL}$. Cutoff values at the 99th percentile are $3.8 \mathrm{ng} / \mathrm{mL}$ for women and $4.4 \mathrm{ng} / \mathrm{mL}$ for men. The coefficients of variations for CK-MB mass, as specified by the manufacturer, are $3.91 \%$ at $3.55 \mathrm{ng} / \mathrm{mL}$ and $3.67 \%$ at $80.16 \mathrm{ng} / \mathrm{mL}$.

\section{Definition of CABG-related MI}

According to the Third Universal Definition [1], MI type $\mathrm{V}$ is defined as an elevation of more than 10 times the 99th percentile during the first $48 \mathrm{~h}$ after CABG. Patients with normal baseline cTnI concentrations plus any of the following criteria were considered to have experienced an MI: (1) new pathologic $Q$ waves or new left bundlebranch block (LB-BB), (2) angiographically documented new graft or new native coronary occlusion, or (3) imaging evidence of new loss of viable myocardium or new regional wall motion abnormality.

\section{Electrocardiograms}

Twelve-lead electrocardiograms (EKG) were obtained from each patient immediately before and $6,12,24$, and $36 \mathrm{~h}$ after CABG. For the identification of new $\mathrm{Q}$ waves, we used the Minnesota code, which is used extensively in epidemiology studies and large-scale clinical trials [13].

\section{Ethics committee approval}

All patients provided written informed consent and were assigned to a treatment group. The Ethics Committee of the Heart Institute of the University of São Paulo Medical School, São Paulo, SP, Brazil, approved the trial. All procedures were performed in accordance with the Declaration of Helsinki.

\section{Statistical analysis}

Values are expressed as mean and standard deviation or median and interquartile range, as appropriate. The paired-sample $t$ test and the unpaired-sample $t$ test were used to compare means within the study group or between subgroups. The chi-square and the Fisher exact tests were used for comparison of discrete variables. Continuous variables without normal distribution were compared using the Mann-Whitney $U$ test, and correlation between such variables was made with the Spearman rank test. Values of $p<0.05$ were considered statistically significant.

\section{Results}

Between March 2012 and April 2014, 326 consecutive patients who met the inclusion criteria were screened. Of these patients, 107 (32.8\%) were excluded (Fig. 1). Of the 219 remaining patients, 148 were referred for CABG (75 $\mathrm{ONCAB}$ and 73 OPCAB [off-pump coronary artery bypass]), and 71 patients were referred for PCI (percutaneous coronary intervention). Of the 75 ONCAB patients enrolled in this study, 21 were excluded and 54 completed the study protocol. These 54 patients had no evidence of MI on CMR assessed by LGE. The main reasons for exclusion of the patients are presented in Fig. 1.

The clinical, demographic, and angiographic characteristics are summarized in Table 1 . The mean age was $61.3( \pm 8.3)$ years, and $39(72.2 \%)$ were male. In addition, $24(44.4 \%)$ patients had a diagnosis of type 2 diabetes mellitus and 13 (24.1\%) had a history of myocardial infarction. Regarding smoking, 18 patients (33.34\%) stopped smoking during the inclusion period of the study. The angiographic screening showed that 17 (31.5\%) patients had stenosis of the left main coronary artery, 43 (80\%) had obstructive lesions in 3 epicardial branches, and 11 (20.4\%) had a concomitant bi-arterial obstructive pattern. Additionally, the mean SYNTAX Score was 28. Anginal symptoms were present in 47 (87\%) patients, and $15(27.8 \%)$ had grade III angina, according to the 


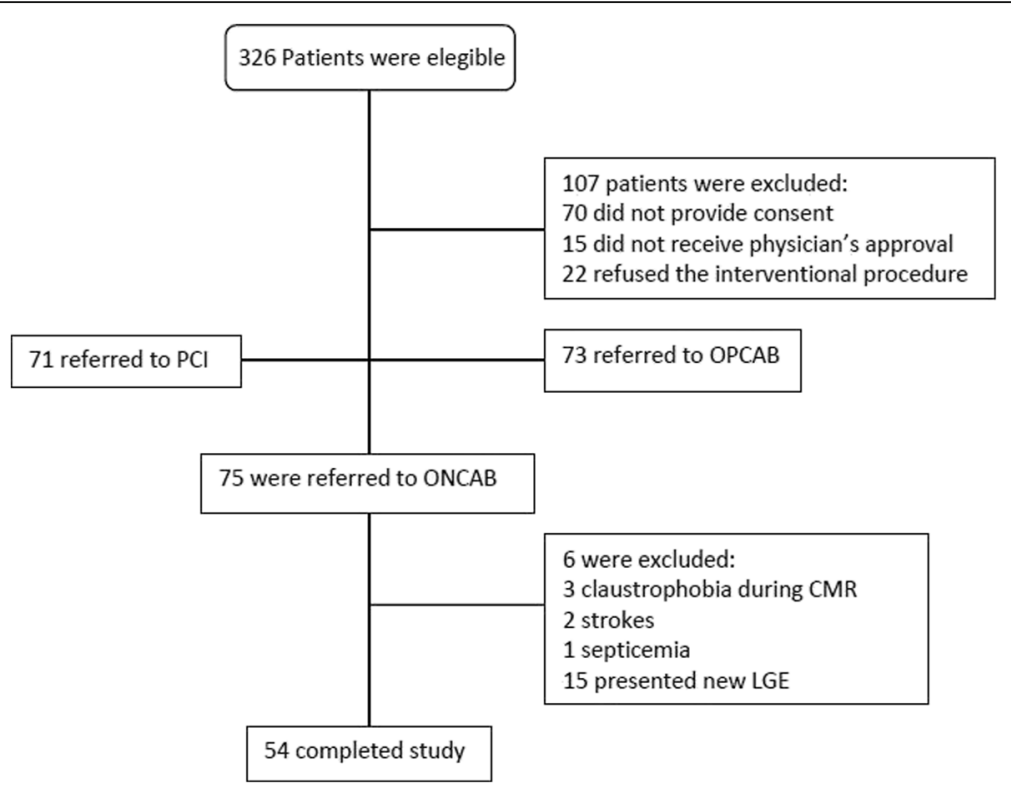

Fig. 1 Consolidated Standards of Reporting Trials (CONSORT) diagram. (CMR = cardiac magnetic resonance; LGE = late gadolinium enhancement; $\mathrm{ONCAB}=$ on-pump coronary artery bypass; $\mathrm{OPCAB}=$ off-pump coronary artery bypass; $\mathrm{PCl}=$ percutaneous coronary intervention)

Table 1 Clinical, demographic and angiographic characteristics of study population

\begin{tabular}{ll}
\hline & $N=54$ \\
\hline Age, $y$ & $61.3 \pm 8.3$ \\
Male, $n \%$ & $39(72.2)$ \\
Diabetes mellitus, $n$ (\%) & $24(44.4)$ \\
Hypertension, $n$ (\%) & $48(88.9)$ \\
Current smoker, $n$ (\%) & $6(11.1)$ \\
Former smoker, $n$ (\%) & $18(33.3)$ \\
Previous myocardial infarction, $n$ (\%) & $13(24)$ \\
Angina, $n$ (\%) & $47(87)$ \\
Angina CCS III-IV, $n$ (\%) & $15(27.8)$ \\
Total cholesterol, mg/dL & $161 \pm 42.4$ \\
LDL cholesterol, mg/dL & $93 \pm 37$ \\
HDL cholesterol, mg/dL & $39 \pm 13$ \\
Triglycerides, mg/dL & $160 \pm 148$ \\
Left main disease, $n$ (\%) & $17(31.5)$ \\
Double-vessel disease, $n$ (\%) & $11(20.4)$ \\
Triple-vessel disease, $n$ (\%) & $43(80)$ \\
SYNTAX Score, & $28 \pm 10$ \\
Ejection fraction, median \% & $66 \pm 8.6$ \\
\hline$N$ Number of patients, CCS Canadian Cardiovascular Society, LDL low-density \\
lipoprotein, HDL high-density lipoprotein
\end{tabular}

Canadian Cardiovascular Society (CCS) scale. Left ventricular ejection fraction was assessed by CRM performed before the procedure and averaged $66 \pm 8.6$ (Table1).

\section{Cardiac biomarkers}

The median value of troponin peak was 3.15 (2.0 to 4.9) $\mathrm{ng} / \mathrm{mL}$, which corresponds to 78.7 times the 99th percentile. Two (4\%) patients had elevation just above the 99th percentile, and 52 (96\%) remaining patients had elevation above 10 times the 99th percentile. There were no patients with a cTnI value below the 99th percentile after the surgical procedure (Fig. 2A).

Regarding CK-MB peak, the median value was $23.0 \mathrm{ng} / \mathrm{mL}$ (14.2 to $38.3 \mathrm{ng} / \mathrm{mL})$. Additionally, 41 (76\%) patients had elevation above the 99 th percentile, and 13 (24\%) had an increase higher than 10 times the 99th percentile (Fig. 2B).

The pattern of cTnI elevation in each moment of evaluation after surgery is shown in the chart below (Fig. 3). Values for cTnI above 10 times the 99th percentile are constant over the measurement time in almost the entire sample.

The comparisons of the levels of cTnI in the different periods after the procedure showed a statistically significant difference, $p<0.001$ in all groups.

The pattern of CK-MB elevation in each moment after surgery is shown in the chart below, respectively (Fig. 4). Only a small part of the sample reached values above the 99th percentile. 


\section{Troponin}

\section{Between 1 to $10 \times 99$ th percentile \\ m $>10 \times 99$ th percentile}

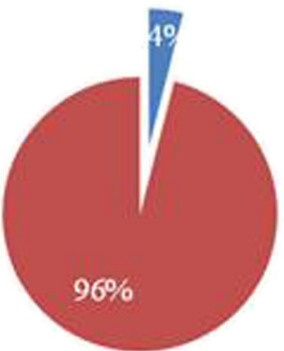

\section{CK-MB}

Between 1 to $10 \times 99$ th percentile

$=10 \times 99$ th percentile

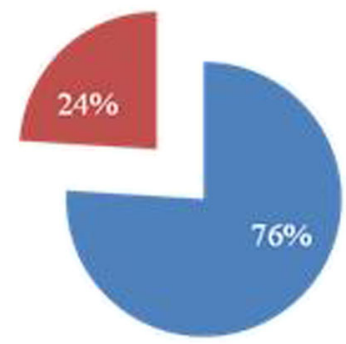

Fig. 2 Percentage of patients with elevated biomarkers according to cutoff levels with no evidence of LGE on CMR

The comparisons of CK-MB values in the different periods after the procedure showed statistically significant differences, $p<0.001$ in both groups.

\section{Electrocardiogram}

All of the 54 patients who were free of late enhancement on the CMR after the procedure and who were selected for this study underwent ECG at entry and sequentially. None of them had a new bundle-branch block, ischemic ST-segment, new pathologic Q wave conduction disorders, or a new $\mathrm{Q}$ wave after the procedure.

\section{Renal function}

All patients had preserved glomerular filtration rate on admission. Sequential measures of renal function indicated that no loss of this function occurred.

\section{Discussion}

In this prospective trial based on current guidelines, we found distinct results when cTnI and CK-MB were simultaneously analyzed in patients after surgical myocardial procedures. All patients had elevated cTnI above the 99th percentile after surgery, with the majority having more than 10 times, reaching an average of 70 times this threshold. Conversely, we found the release of CK-MB predominantly below the recommended threshold of 10 times the 99th percentile. Therefore, our findings conflict with the recommendations of the 2012 European Society of Cardiology/ American College of Cardiology/American Heart Association/World Heart Foundation Joint Task Force for the diagnosis of myocardial infarction after surgical revascularization.

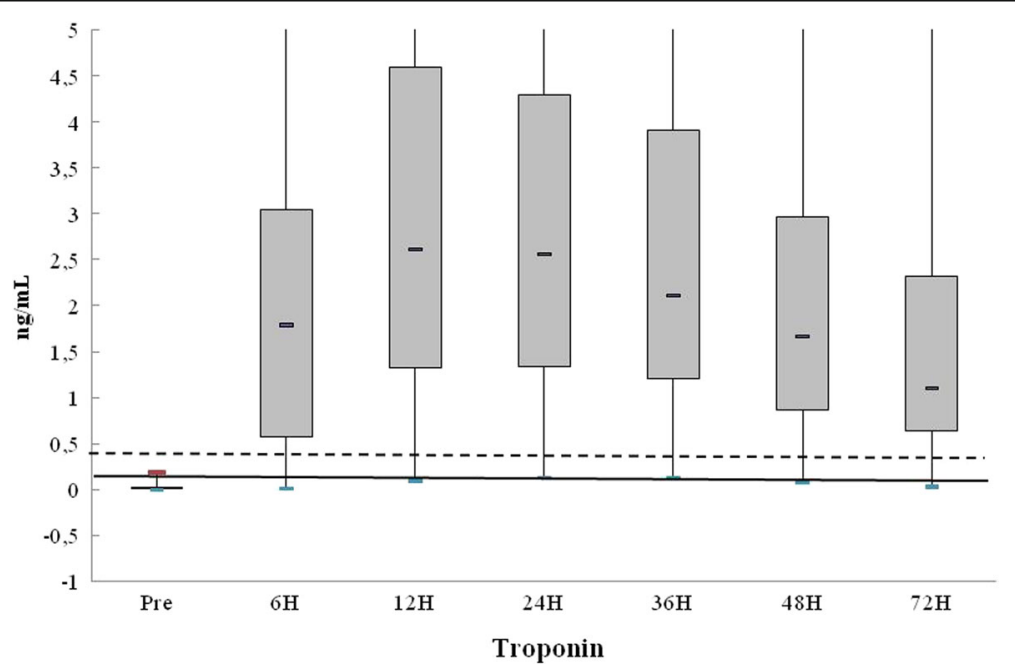

Fig. 3 Distribution of cTnl before and after surgical procedure. Continuous line shows the 99th percentile and dashed line 10 times the 99 th percentile 


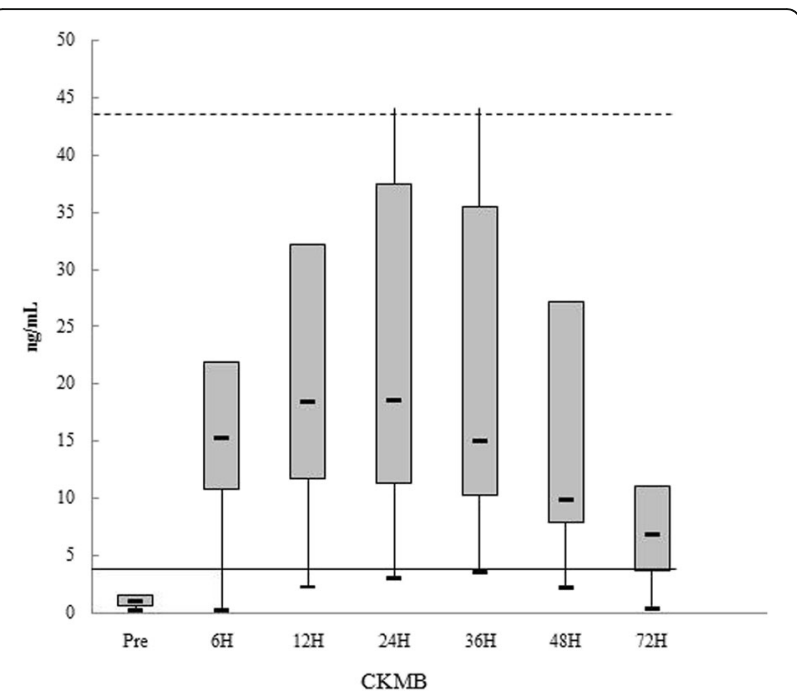

Fig. 4 Distribution of CK-MB before and after surgical procedure. Continuous line shows the 99th percentile and dashed line 10 times the 99th percentile

In this scenario, the EKG remained similar before and after interventions independently of the release of biomarkers. In addition, CMR likewise remained unchanged, without a new delayed enhancement after the procedure.

Over the last decade, elective CABG has progressed to a very standardized and safe surgical procedure with low mortality and low rates of myocardial events [14]. Thus, the present study focused on the possible reasons why our patients, after ONCAB, had elevated troponin above MI levels without the appearance of late enhancement in cardiac magnetic resonance imaging. As we know, perioperative elevation of specific cardiac biomarkers may be due to MI, but may also be associated with routine cardiac surgical procedures.

A study aimed at identifying the release of biomarkers following myocardial revascularization conducted by Pegg et al. [15] identified excessive troponin release in the absence of late enhancement by CMR. On the other hand, they noted that CK-MB behaved as foreseen by the current guideline. Thus, their results confirm the findings of the present study. Likewise, Van Gaal et al. [16], in a study comparing cTnI elevation and appearance of new late enhancement in CMR after CABG, found late enhancement in $28.1 \%$ of their patients. However, troponin elevation was found in $100 \%$ of patients based on the definition of myocardial infarction by the third Task Force [1]. Similar results were observed by Fellahi et al. [17] who, in an accurate analysis, found $14 \%$ troponin elevation in the absence of late enhancement. This lower percentage of discordance was probably due to the use of less-sensitive troponin assays. Wang et al. [18] applying EKG and echocardiography as the gold standard to detect AMI after CABG identified
$21 \%$ of patients with new regional changes in wall motion on echocardiography without the corresponding change on EKG. The echocardiographic findings were consistent with the Troponin Task Force definition. Conversely, EKG data from our study were consistent with CK-MB release and discordant with troponin release.

The evident release of cTnI in the absence of myocardial necrosis is still questioned in the literature. As a confounding factor, advances in cTnI accuracy after high-sensitivity cTnI onset have been recently observed [18]. It can be postulated that this increase in sensitivity may be related to the power to detect changes in myocyte membrane permeability, which may result from non-physiological intraoperative events, contributing to the increase of cTnI plasma levels in the cytosol even in the absence of necrotic damage (Type 5 troponin elevation) $[11,19]$. Therefore, a possible deleterious effect of extracorporeal circulation may contribute to the occurrence of discrete and "diffuse" myocardial damage. This damage can compromise subcellular structures that are difficult to identify, evidencing a clear limitation of CMR analysis.

With the emergence of high-sensitivity troponins, the relationship between the increase in the sensitivity thereof and the increasing rise in false diagnoses has already been described [2]. Currently, there is extensive discussion among manufacturers about the heterogeneity of their troponin kits and the influence of laboratory practices on the use of these kits. The multiplicity of troponin kits, each having different reference values, which use different reagents, different epitopes to bind antibodies, and different incubation times, leads to great difficulty in finding uniformity in the acquired information and studies [20-22]. Unlike that observed with CK$\mathrm{MB}$, the lack of standardization for calibration of the different tests for assessment of cTnI precludes the establishment of a universal threshold cutoff for the 99th percentile [20].

\section{Clinical implications}

Assuming that cardiac biomarkers have limited diagnostic accuracy in myocardial necrosis, the challenges faced for the establishment of definitive values for the diagnosis of myocardial damage include new cutoff values for cTnI. Furthermore, the diagnosis of this condition cannot be exclusively based on cardiac biomarkers or EKG. It is reasonable to include CMR in the set of tools for the accurate diagnosis of procedure-related myocardial injury.

\section{Conclusions}

In this study, different from CK-MB findings, troponin was significantly increased in the absence of myocardial infarction on cardiac resonance imaging. Thus, CK-MB was more accurate than cTnI for excluding procedure- 
related MI. These data suggest a higher troponin cutoff for the diagnosis of CABG-related MI.

\section{Acknowledgments}

We would like to thank all members of the MASS-V Trial for hard work in putting together all the forces needed to perform this study. This study has been funded partially by the Zerbini Foundation and Fundação de Amparo á Pesquisa do Estado de São Paulo (FAPESP) Number 2011/20876-2. Medical writing support was provided by Ann Conti Morcos during the preparation of this paper, supported by the Zerbini Foundation.

\section{Funding}

Financial support for the present study was provided in part by a research grant from the Zerbini Foundation and also by the FAPESP (2011/20876-2).

\section{Availability of data and materials}

Please contact the corresponding author for data requests.

\section{Authors' contributions}

Contribution of each author: FTCO: concept and design of the manuscript; analysis and interpretation of data; $\mathrm{WH}$; conception and design of this study and final approval of the manuscript; $\mathrm{CHN}$ : conception of this trial; $\mathrm{ACH}$ : interpretation of data; AW: acquisition, analysis, and interpretation of data; LMAC: drafting the article or revising it; RMVM: acquisition and analysis of data; PCR: analysis of data; CAWS: drafting the article or revising it; CLG: analysis of data; EGL: interpretation of data, statistical support; JAFR: design of this study and final approval of the submitted manuscript; RKF: final approval of the submitted manuscript. All authors read and approved the final manuscript.

\section{Ethics approval and consent to participate}

The present study was approved by the Ethics Committee of the CAPPesq REGISTRY ONLINE: 8595/2012 - CAAE: 00686812.3.0000.0068-26,441, in Brazil, and adhered to the Declaration of Helsinki. Additionally, written informed consent was acquired from the patients or the patient's relatives.

\section{Consent for publication}

All authors read and approved the final manuscript for publication.

\section{Competing interests}

The authors declare that they have no competing interests.

\section{Publisher's Note}

Springer Nature remains neutral with regard to jurisdictional claims in published maps and institutional affiliations.

Received: 12 September 2017 Accepted: 7 December 2017

Published online: 29 December 2017

\section{References}

1. Thygesen K, Alpert JS, Jaffe AS, et al. Third universal definition of myocardial infarction. J Am Coll Cardiol. 2012;60(16):1581-98.

2. Swaanenburg JC, Loef BG, Volmer M, et al. Creatine kinase MB, troponin I, and troponin $T$ release patterns after coronary artery bypass grafting with or without cardiopulmonary bypass and after aortic and mitral valve surgery. Clinical Chem. 2001;47(3):584-7.

3. Pichon $\mathrm{H}$, Chocron S, Alwan K, et al. Crystalloid versus cold blood cardioplegia and cardiac troponin I release. Circulation. 1997;96(1):316-20.

4. Mingels A, Jacobs L, Michielsen E, Swaanenburg J, Wodzig W, van DieijenVisser M. Reference population and marathon runner sera assessed by highly sensitive cardiac troponin T and commercial cardiac troponin T and I assays. Clinical Chem. 2009;55(1):101-8.

5. Testa L, Van Gaal WJ, Biondi Zoccai GG, et al. Myocardial infarction after percutaneous coronary intervention: a meta-analysis of troponin elevation applying the new universal definition. QJM. 2009;102(6):369-78.

6. Hueb W, Gersh BJ, Rezende PC, et al. Hypotheses, rationale, design, and methods for prognostic evaluation of cardiac biomarker elevation after percutaneous and surgical revascularization in the absence of manifest myocardial infarction. A comparative analysis of biomarkers and cardiac magnetic resonance. The MASS-V trial. BMC Cardiovasc Disord. 2012;12:65.
7. Wu E, Judd RM, Vargas JD, Klocke FJ, Bonow RO, Kim RJ. Visualisation of presence, location, and transmural extent of healed Q-wave and non-Qwave myocardial infarction. Lancet. 2001;357(9249):21-8.

8. KC W, Zerhouni EA, Judd RM, et al. Prognostic significance of microvascular obstruction by magnetic resonance imaging in patients with acute myocardial infarction. Circulation. 1998;97(8):765-72.

9. Selvanayagam JB, Petersen SE, Francis JM, et al. Effects of off-pump versus on-pump coronary surgery on reversible and irreversible myocardial injury: a randomized trial using cardiovascular magnetic resonance imaging and biochemical markers. Circulation. 2004;109(3):345-50.

10. Mahrholdt H, Wagner A, Judd RM, Sechtem U, Kim RJ. Delayed enhancement cardiovascular magnetic resonance assessment of nonischaemic cardiomyopathies. Eur Heart J. 2005;26(15):1461-74.

11. Porto I, Selvanayagam JB, Van Gaal WJ, et al. Plaque volume and occurrence and location of peri procedural myocardial necrosis after percutaneous coronary intervention: insights from delayed enhancement magnetic resonance imaging, thrombolysis in myocardial infarction myocardial perfusion grade analysis, and intravascular ultrasound. Circulation. 2006;114: 662-9. https://doi.org/10.1161/CIRCULATIONAHA.105.593210.

12. Hudsmith LE, Petersen SE, Francis JM, Robson MD, Neubauer S. Normal human left and right ventricular and left atrial dimensions using steady state free precession magnetic resonance imaging. J Cardiovasc Magn Reson. 2005;7(5):775-82. doi:10.1080/10976640500295516.

13. Mendis S, Thygesen K, Kuulasmaa K, et al. World Health Organization definition of myocardial infarction: 2008-09 revision. Int J Epidemiol. 2001; 40(1):139-46.

14. Gober V, Hohl A, Gahl B, et al. Early troponin T and prediction of potentially correctable in-hospital complications after coronary artery bypass grafting surgery. PLoS One. 2013;8(9):e74241.

15. Pegg TJ, Maunsell Z, Karamitsos TD, et al. Utility of cardiac biomarkers for the diagnosis of type $V$ myocardial infarction after coronary artery bypass grafting: insights from serial cardiac MRI. Heart. 2011;97(10):810-6.

16. van Gaal WJ, Arnold JR, Testa $L$, et al. Myocardial injury following coronary artery surgery versus angioplasty (MICASA): a randomised trial using biochemical markers and cardiac magnetic resonance imaging. Eur Secur. 2011;6(6):703-10.

17. Fellahi JL, Gue X, Richomme X, et al. Short- and long-term prognostic value of postoperative cardiac troponin I concentration in patients undergoing coronary artery bypass grafting. Anesthesiology. 2003;99(2):270-4.

18. Wang TK, Stewart RA, Ramanathan T, et al. Diagnosis of MI after CABG with high-sensitivity troponin T and new ECG or echocardiogram changes: relationship with mortality and validation of the universal definition of $\mathrm{Ml}$. Eur Heart J Acute Cardiovasc Care. 2013;2(4):323-33.

19. White HD. Pathobiology of troponin elevations: do elevations occur with myocardial ischemia as well as necrosis? J Am Coll Cardiol. 2011:57(24):2406-8.

20. Lewandrowski KB. Cardiac markers of myocardial necrosis: a history and discussion of milestones and emerging new trends, Clin Lab Med. 34(1) (2014) 31-41, xi.

21. Araújo MP, Mesquita ET. Avaliação de Marcadores Prognósticos na Síndrome Coronariana Aguda sem Supradesnivelamento do Segmento ST na Sala de Emergência. SOCERJ. 2005:50-6.

22. Ramasamy I. Biochemical markers in acute coronary syndrome. Clin Chim Acta. 2011:412(15-16):1279.

\section{Submit your next manuscript to BioMed Central} and we will help you at every step:

- We accept pre-submission inquiries

- Our selector tool helps you to find the most relevant journal

- We provide round the clock customer support

- Convenient online submission

- Thorough peer review

- Inclusion in PubMed and all major indexing services

- Maximum visibility for your research

Submit your manuscript at www.biomedcentral.com/submit
Biomed Central 